Archives de sciences sociales des religions

139 | juillet - sepembre 2007

Prophètes, messages et médias

\title{
Une retraite de carême sur Internet
}

Isabelle Jonveaux

\section{(2) OpenEdition}

Journals

Édition électronique

URL : http://journals.openedition.org/assr/9533

DOI : 10.4000/assr.9533

ISSN : $1777-5825$

Éditeur

Éditions de l'EHESS

Édition imprimée

Date de publication : 1 septembre 2007

Pagination : 157-176

ISBN : 978-2-7132-2144-6

ISSN : 0335-5985

Référence électronique

Isabelle Jonveaux, "Une retraite de carême sur Internet », Archives de sciences sociales des religions [En ligne], 139 | juillet - sepembre 2007, mis en ligne le 19 novembre 2010, consulté le 01 mai 2019. URL : http://journals.openedition.org/assr/9533 ; DOI : 10.4000/assr.9533 


\section{Isabelle Jonveaux}

\section{Une retraite de carême sur Internet}

Pour la quatrième année consécutive, les frères dominicains du couvent de Lille ont proposé, pour toute la durée du carême 2006, une formule de retraite sur Internet, intitulée Retraite dans la Ville (www.retraitedanslaville.org). Ce site propose des conférences en ligne, les offices du couvent, des forums de discussion pour les internautes, une "foire aux questions ", ainsi qu'un accompagnement spirituel en ligne. Le nombre des inscrits n'ayant cessé d'augmenter depuis quatre ans, la formule semble répondre à une attente : de qui émane-t-elle ?

Cet événement illustre de nombreux aspects de la modernité religieuse : recul des pratiques, individualisation du croire, demande d'émotion à la religion, plausibilité d'un discours religieux dans une société sécularisée. En arrière-plan, se pose la question de la plausibilité aujourd'hui d'une extramondanité religieuse ; si les dominicains proposent une retraite « à domicile ", faut-il en conclure qu'il n'y a plus de sortie du monde possible, qu'il n'y a plus de recherche de salut hors du monde ? Entre aussi en jeu la problématique du virtuel, à laquelle est liée toute pratique sur Internet. Qu'en est-il d'une pratique religieuse sur Internet et que dire d'une pratique religieuse virtuelle, liée à la question de l'impersonnalité et du désengagement qu'elle implique?

Pour cette étude, nous nous appuierons principalement sur une enquête menée sur le site de Retraite dans la Ville, avec la lecture des différentes rubriques ainsi que sur deux entretiens réalisés auprès d'un jeune dominicain, le frère Pascal, un des prédicateurs de la retraite, au début et après la fin du carême. Nous avons pu, aussi, avoir accès aux résultats des enquêtes de fin de retraite 2005 et 2006.

En premier lieu, cette retraite consiste visiblement à amener la religion aux individus qui n'iraient pas, ou plus, spontanément vers elle. Ainsi se vit dans le monde, chez soi, ce qui se vivait auparavant hors du monde (dans les monastères). Devenue intramondaine, cette expérience religieuse peut aussi devenir virtuelle posant de nombreuses questions sur sa légitimité, et sur ses caractéristiques.

\section{Des dominicains confrontés à la modernité}

Le carême chrétien est un temps privilégié de renouvellement et d'approfondissement de la foi, particulièrement adapté à des enseignements, et dans lequel 
s'inscrivent les traditionnelles « conférences de carême ». Il peut donc être l'occasion d'un renouveau ou d'un lancement de la pratique religieuse.

Avec les conférences dans les couvents, l'objectif premier des dominicains était de participer à l'élan d'enseignement lié au carême. L'évolution de la situation religieuse : recul des pratiques régulières, exculturation du catholicisme (HervieuLéger, 2003 : 87) ${ }^{1}$, difficultés d'accès à la religion dans certaines régions où la baisse des vocations laisse de nombreuses églises sans prêtre (Pelletier, 1997) ${ }^{2}$, a conduit les dominicains à utiliser d'autres outils pour se rapprocher de ceux restés loin de la religion. C'est dans cette perspective qu'ils ont créé, à l'occasion du carême de 2003, Retraite dans la Ville sur Internet. Le nombre des visiteurs a très vite augmenté : 5200 inscrits en 2004, 8090 en 2005 et 15500 en $2006^{3}$. L'attrait pour cette formule est donc incontestable, mais il s'agit de savoir qui est le public concerné. Pour les dominicains, elle s'adresse aux «très nombreuses personnes qui n'ont plus aujourd'hui la possibilité de suivre une retraite traditionnelle ; le manque de temps étant souvent un obstacle déterminant. » En réalité, le manque de temps n'est pas une raison suffisante pour expliquer le recul des pratiques mais l'invocation de ce prétexte permet aux individus de déculpabiliser car c'est celui qu'ils se donnent et qui semble reconnu par des « autorités religieuses ». Ce n'est pas qu'ils soient moins religieux, mais les occupations du monde ne leur permettent plus d'avoir une profonde vie religieuse. Ainsi, Retraite dans la Ville propose d'autres outils pour la pratique de carême, destinés à ceux qui ne pratiquent plus régulièrement. Reste à savoir si ce sont bien ces personnes, en marge de l'Église, que ce site est parvenu à rattraper. Pour cela, il faut tout d'abord identifier les non pratiquants et les utilisateurs d'Internet. Cette population des non-pratiquants correspond principalement aux catégories jeunes : 10,0\% des femmes de 15 à 24 ans et $8,9 \%$ des hommes de cette même tranche d'âge disent pratiquer régulièrement quand $26,2 \%$ des femmes de plus de 60 ans donnent la même réponse ${ }^{4}$. Quant à la tranche des 25-34 ans, seuls 10,9\% des femmes et $7,8 \%$ des hommes se disent pratiquants réguliers.

Parallèlement, cette même catégorie des 15-24 ans représente, en juin $2006^{5}$, $24 \%$ des utilisateurs d'Internet; on atteint $49 \%$ si l'on rajoute la catégorie des 25-34 ans alors que les plus de 49 ans n'en représentent que $22 \%$. Internet semble donc être l'outil idéal pour atteindre ces catégories jeunes de la population qui ne pratiquent pas ou peu.

1. «Mais le problème qu'on soulève ici porte plus loin et plus largement : il concerne la possible déliaison de l'affinité élective que l'histoire a établie en profondeur entre les représentations partagées des Français (la "culture" qui leur est commune) et la culture catholique. C'est cette déliaison qu'on s'efforce de cerner en parlant d'exculturation du catholicisme français ».

2. Le nombre de paroisses a été réduit de près d'un tiers entre 1980 et 1993, ce qui entraîne l'absence de messe dominicale dans nombre d'églises.

3. Environ 19000 participants à la fin du carême 2007.

4. Source : INSEE, Enquête permanente sur les conditions de vie, 2003.

5. Source : IPSOS Média juin 2006. 
Cependant, l'utilisation de cet outil dépend aussi des catégories socioprofessionnelles. En effet, en juin 2006, les catégories socioprofessionnelles supérieures représentent $38 \%$ des internautes, ce chiffre étant de $29 \%$ pour les catégories socioprofessionnelles moins élevées ${ }^{6}$. Pour suivre un office en ligne, il faut pouvoir rester longtemps sur Internet, en mettant le son, il est donc plus pratique de le faire chez soi, avec Internet à haut débit, forfait illimité plutôt qu'au bureau ou avec un forfait limité ${ }^{7}$. De plus, le biais technique peut introduire de multiples difficultés que les dominicains tâchent d'évaluer dans les enquêtes de fin de retraite, pour les réduire. Le site doit être d'utilisation facile pour que le degré de compétence en informatique ne soit pas discriminant. Il faut donc prendre en compte des critères sans rapport direct avec la religion.

Internet semble néanmoins être l'un des outils les plus appropriés pour se rapprocher des personnes géographiquement éloignées de la religion par manque de ministres du culte et des personnes les moins pratiquantes correspondant justement aux catégories les plus utilisatrices d'Internet.

Face à la déchristianisation, l'un des enjeux majeurs de l'Église est de proposer un message plausible qui puisse être reçu et accepté dans le cadre de la modernité religieuse. Si les dominicains de Retraite dans la Ville souhaitent atteindre les individus qui sont "à la marge ", il est nécessaire que leur message soit compréhensible et qu'il ne présuppose pas de culture religieuse approfondie, ce qui nécessite une adaptation au langage du siècle, donc une sécularisation du langage religieux.

«On est attentif dans notre site à rejoindre les gens donc à ne pas employer le jargon religieux et à employer leur langage, à transmettre la foi de l'église quand je dis la foi de l'église, en fait, je pense la parole de Dieu, à offrir un espace pour Dieu dans le langage des gens. » (Frère Pascal)

Ainsi, les dominicains prennent acte de ce que Danièle Hervieu-Léger (2003) appelle "l'exculturation du catholicisme », le retrait de la culture catholique de la société. Il fut une époque où les références catholiques étaient comprises de tous, ce n'est plus le cas aujourd'hui, parmi les baptisés eux-mêmes ${ }^{8}$. Le médium utilisé permettrait aussi d'atteindre un public plus vaste, sa plus grande plausibilité étant due au fait qu'Internet est inscrit dans la modernité, qu'il en est un acteur essentiel, et qu'ainsi, une pratique religieuse par cet outil permet à l'individu de se croire dans le siècle, davantage qu'en entrant dans une église 9 . De la traditionnelle conférence de carême à l'ambon d'une église à la conférence de carême sur Internet,

6. Source : IPSOS Média juin 2006 (CSP+ et CSP ).

7. Sur le forum, un internaute fait ses adieux car il a utilisé tout son forfait.

8. Par exemple, à la question «Savez-vous quelle fête correspond à la descente de l'Esprit saint sur les Apôtres ? ", 33 \% seulement des catholiques répondent la Pentecôte. Le Monde des Religions, janvier-février 2007.

9. «C'est super votre retraite. Pour la première fois, j'ai l'impression d'être catholique, sans être ringard. Vous me réconciliez avec moi même, homme dans la vie moderne, et croyant » (citation de la rubrique "témoignages »). 
le médium s'est sécularisé, sortant d'une sphère purement religieuse. Les sujets abordés se veulent actuels, pour qu'ils puissent véritablement " parler » aux gens. D'où le thème de l'année 2006 : "Chaque jour j'entends dire : où est-il ton Dieu ? " ${ }^{10}$, qui, dans le contexte du psaume, invite le croyant à chercher Dieu dans la société contemporaine malgré son refus, ce qui peut fort bien s'appliquer aujourd'hui. Les organisateurs sont très attentifs, notamment grâce à l'enquête soumise aux participants en fin de retraite, à rendre leurs conférences accessibles aux gens dans leur quotidienneté :

«Alors il s'agit de savoir par exemple si les gens ont trouvé les conférences ou bien trop longues, ou bien trop déconnectées de leur vie quotidienne, "oui, c'est bien tout ça, mais ça c'est du blabla catho, en quoi tout ça, ça me concerne moi dans ma vie ?”, voilà. Et comme nous, notre but, c'est quand même de rejoindre les gens dans leur vie, dans leur chair, heu... » (Frère Pascal)

D'après l'enquête 2006, des personnes réclament même une inscription dans l'actualité de la société, certains regrettant, par exemple, qu'il n'ait été fait aucune mention de la crise du CPE, survenue au même moment ${ }^{11}$ témoignant bien que le «siècle gagne du terrain sur le religieux [...], l'activité et la pensée religieuse tendent à s'aligner sur l'activité et la pensée profane » (Isambert, 1976). Les croyants attendent de la religion une clé de lecture de la société toujours plus complexe et dénuée de cadres, plutôt que l'annonce d'un salut extramondain.

\section{Internet au secours de l'extramondanité impossible}

Si les dominicains proposent aujourd'hui une retraite à vivre chez soi, est-ce parce que la sortie du monde est devenue impossible ? La présentation de Retraite dans la Ville tend à prouver qu'elle devient, effectivement, de plus en plus difficile pour les individus. Manque de temps, préoccupations multiples, liens de communication retiennent dans le monde.

Pourtant, Danièle Hervieu-Léger constate qu' " au moment où l'étrangeté de la vie monastique et sa distance à la culture moderne semble n'avoir jamais été aussi grandes, la circulation des visiteurs et des "retraitants" a rarement été aussi intense » (1986: 9). Peut-être faudrait-il alors distinguer ce qu'elle appelle une «variante sécularisée thérapisée de la retraite monastique » ${ }^{12}$ de la traditionnelle retraite monastique comme véritable pratique religieuse. La première ne serait qu'une cure, physique ou spirituelle, quand la seconde serait une sortie du monde,

10. Psaumes 41,4.

11. Citation d'une réponse à la question ouverte du sondage 2006 : «Faire le lien entre les Écritures et la vie. Il est choquant de ne trouver aucune réflexion, aucune prière avec le mouvement des étudiants et des salariés contre le CPE en plein moment de carême. Ce mouvement dit quelque chose à l'Église quand les jeunes se préoccupent de leur avenir, de leurs conditions de vie..." .

12. Op. cit., p. 10. 
un retrait au désert comme pratique religieuse. Dans l'une, on se retire pour se chercher, dans l'autre, on cherche Dieu. Ainsi, l'extramondanité sécularisée est pleinement à la mode tandis que l'extramondanité religieuse perd sa signification. Car la question est bien celle de l'existence aujourd'hui de possibilités d'extramondanité, entendue comme l'attitude de celui qui sort du monde pour chercher Dieu, dans une vision plus large du concept de Max Weber pour lequel, l'extramondanité ne s'applique qu'aux religions de salut et non aux états humains.

Il est, à ce sujet, révélateur de constater la disparition de la thématique du désert, image traditionnelle d'une retraite et particulièrement d'une retraite $\mathrm{du}$ carême sur le modèle de Jésus se retirant quarante jours au désert.

« On voit cette année qu'on abandonne la thématique du désert, qui était avant le fil directeur de Retraite dans la ville et qui est la thématique traditionnelle de la retraite, aller au désert. Aujourd'hui nous avons abandonné cette idée du désert qui, de fait, ne parle plus à beaucoup de gens, et sur notre logo on voit des immeubles et des maisons, donc une ville. » (Frère Pascal)

Abandonner l'image du désert et choisir celle de la ville, c'est bien ramener dans le monde ce qui se passait habituellement hors de lui. Ce mouvement fut aussi celui de la Réforme contre le monachisme extramondain (Weber, 2003). Dire que la «thématique du désert ne parle plus à beaucoup de gens ", c'est reconnaître les difficultés à sortir du monde et surtout, c'est l'accréditer en acceptant de s'y adapter. Plutôt que de relancer ce mouvement de sortie du monde, les dominicains préfèrent « intramondaniser » la religion catholique, ou ses pratiques, pour conserver une plus grande plausibilité.

Non seulement, cette retraite se déroule dans le monde, contrairement aux retraites traditionnelles, sans déplacement, mais plus encore, elle se déroule sur le lieu du paroxysme de la communication et de l'information mondiales. Le défi pour les dominicains, est donc, comme le dit le Frère Pascal, de créer un espace pour la parole de Dieu sur Internet, qui peut néanmoins permettre à l'individu de reconstituer certaines conditions caractéristiques d'une retraite.

Dès le XVII ${ }^{\mathrm{e}}$ siècle apparaît un mouvement d'individualisation de la relation à Dieu qui annonce et prépare la modernité religieuse. Ce mouvement s'intensifie au $\mathrm{XX}^{\mathrm{e}}$ siècle avec une véritable individualisation du croire qui débouche sur ce que Danièle Hervieu-Léger appelle le « bricolage » des croyances.

Retraite dans la Ville, qui se présente comme un lieu spirituel ouvert à tous, dans la liberté, sans condition d'accès ni rite d'initiation (il n'est pas nécessaire d'être baptisé par exemple), semble répondre à cette individualisation de la relation religieuse. Pour recevoir les messages de prière tous les jours, il faut naturellement s'inscrire au préalable, mais cette inscription est libre, peu de renseignements obligatoires sont demandés, et de surcroît gratuite. Pour leur part, les méditations sont consultables sur le site sans inscription, ce qui peut, selon les dominicains, convenir aux jeunes qui préfèrent ne pas s'inscrire. L'accès est libre dans l'espace et libre dans le temps. Contrairement à une messe ou à des vêpres, auxquels on 
assiste physiquement, les prières peuvent se lire à n'importe quel moment et les vêpres peuvent être écoutées à toute heure de la journée et de la nuit. Ainsi, il s'agit, comme l'affirme le dossier de presse, d' " une démarche souple » qui répond en ce sens aux désirs religieux de la modernité, un service religieux ponctuel, sans engagement.

Comme le dit le Frère Pascal, le but de cette retraite est de "ne pas se replier sur ceux qui sont là mais d'aller vers les autres. ». Il faut alors identifier quels sont ces " autres ", ceux qui ne sont pas "déjà là ", et voir ensuite si cet événement peut effectivement les toucher.

Retraite dans la Ville, par son médium qui amène, pour ainsi dire, « la religion à domicile ", s'adresse pour une part aux individus géographiquement éloignés des lieux de dispense de la religion. Par exemple, aux expatriés heureux de pouvoir suivre une retraite en français - selon les dominicains, pas moins de quarantecinq pays seraient représentés - mais aussi, et surtout, à tous ceux vers qui l'Église ne va pas (ou plus). Car en réalité, c'est aussi l'Église qui s'éloigne des individus et non pas seulement les individus qui s'éloignent de l'Église. La retraite sur Internet vient alors jusque chez les individus géographiquement éloignés de la religion, ce qui ne signifie pas forcément que leur éloignement soit également spirituel. De nombreux témoignages du forum viennent corroborer cette hypothèse, pour qui les vêpres en ligne permettent d'assister - virtuellement - à un office. Ces personnes ne sont donc pas les habitants des villes tel que le logo et le nom de la retraite le laisseraient supposer, mais au contraire, ceux des campagnes retirées. Cette retraite en ligne, «à domicile » permet aussi d'atteindre ceux qui souffrent d'un handicap les empêchant d'aller vers la religion. Des messages dans la rubrique " témoignages » le confirment :

« Un grand merci pour l'aide que vous apportez aux sourds qui peuvent lire ne pouvant aller aux conférences de carême car il n'y a personne pour les aider. C'est vraiment très heureux. »

Ou encore :

"Je découvre ce site avec joie et beaucoup de bonheur; je vais de ce pas le faire connaître à mon amie. Elle est handicapée et "cloîtrée" chez elle, Internet est une évasion extraordinaire pour elle. »

Ces catégories n'étaient pas les premières visées, mais ces témoignages confirment la capacité des religieux à mobiliser les outils de la modernité au service de leur message.

Enfin, la catégorie que visent particulièrement les dominicains est celle des individus spirituellement éloignés de l'Église, principalement les jeunes, en dessous de 25 ans ou même de 34 ans. Ce but est illustré par le logo de la retraite :

"Lorsqu'on arrive sur le site, on voit effectivement un jeune homme qui arrive par la gauche de l'écran et qui arrive devant l'affiche de Retraite dans la ville, devant le logo. Oui, c'est (pause) ça représente quelqu'un du public qu'on pourrait viser, qu'on 
pourrait rechercher c'est-à-dire, là c'est un jeune qui doit avoir une vingtaine d'années, qui sans doute ne se pose pas de questions religieuses, et qui, voilà, regarde, avec intérêt, ce qu'on lui propose. » (Frère Pascal)

Retraite dans la ville par son arrivée "à domicile " permettrait ainsi à ceux qui n'oseraient pas faire une démarche institutionnalisée de créer ou recréer un lien avec la religion. Pour savoir s'ils y parviennent, les dominicains envoient à la fin de chaque retraite un questionnaire pour connaître les caractéristiques des retraitants. D'après les sondages des années 2005 et 2006, on observe que, manifestement, le désir d'atteindre les jeunes éloignés de la religion n'est pas atteint. En 2005, le profil type du retraitant est la mère de famille au foyer, qui allume son ordinateur après avoir accompagné les enfants à l'école, ou une femme entre 50 et 65 ans, ou enfin un actif entre 40 et 50 ans qui a accès à Internet sur son lieu de travail. Cela ne correspond ni aux catégories initialement visées ni à celles identifiées comme les plus utilisatrices d'Internet, mais bien à celles des pratiquants réguliers. D'après ces enquêtes, la majorité $(91 \%)^{13}$ des individus est proche ou très proche de l'Église ${ }^{14}$ alors que cette retraite était, en fait, destinée à ceux qui sont "en marge ". Toutefois, on peut lire ces données de manière inversée et mettre en relief les $8 \%$ restants pour prouver qu'il y a effectivement des inscrits qui se disent par ailleurs « éloignés » ou "très éloignés » de l'Église. Si l'on compare les deux dernières années, on passe de 7,6 \% à 8,2 \% de personnes " très éloignées " ou "éloignées ": on note donc une légère augmentation mais, globalement, les proportions restent les mêmes. On peut citer ce témoignage d'une personne «éloignée » qui participe à la retraite :

"Merci de venir à nous qui ne venons pas facilement vers l'Église, vers les diverses communautés qui la constituent. Appartenant à la génération 68 qui a pris le grand large et tente de revenir sur la pointe des pieds, ce n'est pas facile de trouver ses marques, de ne pas se sentir perdu ou coincé entre l'ancien et le nouveau. Vous représentez un accueil, une présence, une ouverture, une parole de lumière, c'est une grande joie de vous retrouver chaque matin. Merci. » (rubrique témoignages)

De même, pour les jeunes, 4,1 \% d'entre eux, seulement, ont moins de 25 ans en 2006, alors que c'était l'une des catégories les plus visées, notamment du fait du médium utilisé. Finalement, le public de Retraite dans la Ville se superpose à celui des pratiquants et non pas à celui des internautes. Pour les années à venir, le frère Pascal insiste sur le fait qu'il faut " essayer d'atteindre un public plus jeune, plus éloigné de l'Église ${ }^{15}$. Cette retraite veut s'adapter aux différents parcours et configurations spirituelles peu traditionnels d'où l'importance des

13. Le sondage 2007 confirme cette tendance : $93 \%$ se disent proches de l'Église ou engagés.

14. D'après l'enquête sur la retraite 2005, sur 3240 réponses au questionnaire, 1765 se disent très proches de l'Église, 1204 proches, 236 éloignés et 35 très éloignés, et 305 sont religieux ou prêtres. En 2006, 38 se disent très éloignés, 315 éloignés, 1553 proches, 2113 engagés et 327 sont religieuses, religieux ou prêtres.

15. En réalité, le but n'est pas encore atteint en 2007 : à peine $4 \%$ des participants se disent éloignés de l’Église. 
citations dans les média à grande audience ${ }^{16}$ qui concernent des publics extrêmement diversifiés sans déterminants religieux. Les résultats du sondage mettent néanmoins en lumière une contradiction entre les catégories initialement visées et celles qui sont réellement touchées.

\section{Quelles pratiques religieuses pour Internet ?}

Dans le cadre d'une sociologie des pratiques religieuses, il serait intéressant de pouvoir déterminer quelles sont les pratiques qu'Internet favorise. Autrement dit, remplace-t-il simplement un livre, Bible ou livre de prière, ou bien remplitil une fonction spécifique irréductible à aucune autre ? Et dans ce cas, quel est ce rôle? Qu'est-ce que les retraitants utilisent du site, et combien de temps y passent-ils. Car un "passage » sur le site ne peut pas être considéré comme un véritable acte religieux ${ }^{17}$. L'enquête 2006 , et plus particulièrement la question portant sur l'office, aident à préciser s'il est seulement écouté en bruit de fond ou s'il fait l'objet d'un véritable temps de prière. Sur les personnes qui disent avoir écouté régulièrement les vêpres, 57 \% répondent avoir « pris un temps de prière en suivant le texte sur l'écran ". Internet peut-il devenir le lieu d'une véritable pratique religieuse ? D’après des témoignages, certains aménagent une sorte d'autel, avec bougies, pour créer un espace religieux autour de leur ordinateur ${ }^{18}$. Il semble donc qu'Internet puisse créer une présence religieuse de substitution, mais c'est une question qu'il faut sans doute encore approfondir.

Grâce à l'outil informatique, le " temps pour Dieu » peut se prendre n'importe où et des témoignages montrent que beaucoup des actifs participant à cette retraite lisent les méditations au travail. La retraite spirituelle se déroule donc sur le lieu (et peut-être le temps !) de travail. Il y aurait sans doute beaucoup à dire sur ce sujet, à l'aune des grands débats sur l'opposition entre contemplation et travail (Weber, 2003) à partir de la Réforme protestante où l'ascèse intramondaine se vit dans et par le travail. Cependant, il n'y a ici aucun lien explicite entre travail et prière. Reste donc à savoir si ces personnes le font par choix ou si c'est le seul endroit où elles ont accès à Internet. Si c'est par choix, est-ce en vue de sanctifier la journée de travail, de lui donner un sens religieux ou par simple opportunisme?

16. Pour 2005 : journal de $20 \mathrm{~h}$ de France 2, 6 minutes de M6, informations sur France Inter, France Bleu, dépêche AFP, etc.

17. "Le site a été visité plus de 154100 fois [entre le début de la retraite et le 19 mars]. Entre 15 et 30 minutes pour 20766 visites (soit 13,5\%). Dont 194 visites depuis Rome (dont, on peut le supposer, le Vatican !). 2,5\% de ces visiteurs ont visité le site pendant une durée de plus d'une demi-heure. » Pour toute la durée de la retraite, on compte 255000 visites du site. Frère Pascal David, "Rapport de fin de charge ", Retraite dans la Ville, 2007.

18. "C'est vraiment un moment de bonheur pour moi chaque jour de me mettre devant mon ordinateur, de lire la prière du jour et d'écouter un office. J'installe une icône et une bougie et j'ai vraiment l'impression d'être en communion totale avec les frères dans leur couvent et avec tous les autres chrétiens qui prient en même temps»(rubrique témoignages du site). 


\section{Le forum : de la communauté d'identité à la communauté d'émotion}

Parmi les participants à la retraite, les dominicains identifient une catégorie particulière : les internautes du forum, dont les besoins religieux semblent être sensiblement différents des autres retraitants. Ce groupe est intéressant car il reproduit de nombreuses caractéristiques de la modernité religieuse. Parallèlement à l'individualisation du croire, la modernité religieuse fait naître un besoin de communautarisation que Retraite dans la Ville illustre de multiples façons malgré sa nature virtuelle. En plus de la communauté bien réelle des dominicains, se construit par le forum une communauté d'identité qui devient aussi une communauté émotionnelle. Les internautes, peu nombreux, se retrouvent d'une année sur l'autre et finissent, au fil des jours et des messages, par se connaître. Cette communauté est, d'abord, une communauté d'identité, née de la joie de se retrouver entre chrétiens, d'échanger sur des expériences liées à la foi. Les parcours spirituels sont divers, plus ou moins proches de l'Église, mais où l'on s'accompagne, se soutient pour parvenir au même but. Les internautes du forum mettent eux-mêmes en scène cette construction de la communauté qui apparaît à la lecture de leurs messages :

"Merci et quel bonheur de voir des liens se tisser entre nous. "

Ou encore :

« À travers toi et beaucoup ici : Clématite, Isabelle et d'autres encore dont j'oublie certainement le nom, je redécouvre ici un bout de ce que Thérèse appelle la "grande communauté", celle à laquelle je continue à dire que je crois, l'Église de Jésus. Merci d'avoir été pour moi et tant de chrétiens homosexuels des témoins de l'amour de Dieu. »

Cette communauté d'identité veut aussi se poursuivre en communauté de prière, non seulement pour le temps du carême mais pour le reste de l'année. Ainsi, nombreux sont les messages dans lesquels l'auteur annonce qu'il va prier pour celui à qui il répond ou pour les dominicains qui les accueillent. De même, il est proposé que les internautes récitent un Notre Père à 19 heures toute l'année pour créer une communion de prière. Très vite, ce forum permet la formation d'une communauté émotionnelle. L'émotion se vit dans le témoignage que chacun apporte. On peut citer notamment le témoignage poignant des homosexuels catholiques qui peinent à trouver leur identité. Un grand nombre de messages remercient pour la beauté et la grandeur des mots utilisés. Cette configuration se retrouve pour ces trois débuts de message qui suivent le récit d'une expérience particulière de prière dans une église :

" Je suis une nouvelle mais je trouve ton histoire assez touchante. »

"Ton histoire est très belle et très touchante à la fois. »

"J'ai trouvé ton témoignage très touchant. »

Il arrive que sur certains sujets, le ton monte et se succèdent alors des messages de paix, ou quelqu'un menace de quitter le forum et d'autres tentent de le ramener. 
Chacun se dévoile, de manière anonyme, jusqu'au plus profond de lui-même et on par rivalise dans la force d'émotion des messages où le ton avoisine le pathétique. Citons cet exemple, dans la rubrique " cancer »:

"Sandra, ma sœur, permets-moi de t'appeler ma sœur. »

L'apothéose de l'émotion et du pathétique est atteinte dans une rubrique qui invite tous les internautes du forum à écrire à Nathalie $\mathrm{D}$, hospitalisée pendant la semaine sainte, pour lui montrer qu'ils continuent de penser à elle. La moitié seulement des messages est adressée au bon rétablissement de Nathalie, les autres saluent l'initiative et s'extasient de la beauté des pensées, de la force communautaire et émotionnelle engendrée, ou parlent d'autres malades qu'ils connaissent et accompagnent. Ce type de situation où chacun essaie de raconter l'histoire la plus touchante possible conduit à une surenchère de l'émotion. L'anonymat du pseudonyme est alors suspendu et beaucoup signent de leurs vrais noms.

Enfin, dans d'autres rubriques, certains avouent avoir pleuré à la lecture de messages :

"Chère Clématite, merci du fond du cœur d'avoir accueilli dans votre prière mon mari ; je n'oublie pas que vous nous avez aussi confié votre mari à notre prière. Comptez aussi sur ma prière. Cher Jean, merci du fond du cœur de m'accueillir avec mon mari dans votre prière, j'en suis touchée et honorée. Des larmes enfouies au fond de mon cœur sont montées à mes yeux en lisant vos mots ce matin. Merci.»

La religion n'est alors plus le premier déterminant de la communauté, elle est certes le référent de tous les sujets abordés mais ce qui prime, dans certaines rubriques, c'est l'émotion que font naître les confidences de chacun. D'où l'interrogation des dominicains sur la pertinence du forum sur un tel site, alors que des internautes dénoncent ces " dérives émotionnelles " ${ }^{19}$. Car il existe différents forums de discussions chrétiens et Retraite dans la Ville ne veut pas être l'un d'eux mais le lieu d'une retraite. De plus, les participants, en vérité peu nombreux, une quarantaine environ, bien que très actifs et selon les dominicains, sont des habitués des forums sur Internet. En ce sens, ils correspondent bien à la population des utilisateurs d'Internet, mais ne profitent pas nécessairement d'autres services offerts par Retraite dans la Ville. En outre, l'affluence des messages, dénoncée par certains internautes, remet en cause le projet des dominicains de proposer autre chose que la rapidité et la profusion incontrôlables caractéristiques d'Internet. Enfin, les sujets des messages ne correspondent pas exactement à ce que les dominicains souhaiteraient. Certes, ils veulent proposer un espace de parole ouvert à tous, mais s'interrogent sur la pertinence de ces sujets de société dans un espace de retraite. Il n'est donc pas assuré que le site 2007 propose ce forum, ou du moins, sous cette forme ${ }^{20}$.

19. Citons, par exemple : "Mais ce qui me choque c'est de voir que ce cadre devient un lieu où les gens se soutiennent et se consolent mutuellement au lieu de chercher la sagesse de Dieu. »

20. Effectivement, le forum a été supprimé en 2007. 
Avec ces dérives, ce forum illustre les dangers que peut représenter la liberté d'Internet et, par-là même, les limites de cet outil. Car, non seulement, les dominicains ne peuvent pas en choisir les consommateurs, s'adressant véritablement, et uniquement peut-être, à ceux qu'ils souhaiteraient atteindre, mais en plus, ils ne peuvent contrôler tout ce qui s'y déroule. Se pose, ici, la question centrale de la virtualité.

\section{Le bricolage des croyances}

L'individualisation du croire, évoquée précédemment, débouche sur le bricolage des croyances et des pratiques où chacun constitue sa religion à partir des éléments auxquels il adhère, laissant de côté ceux qui ne lui conviennent pas. Un événement religieux tel que Retraite dans la Ville témoigne de ces bricolages non seulement par les propos des internautes sur le forum mais aussi parce que sa souplesse permet une grande liberté dans les pratiques ${ }^{21}$.

Le forum propose ainsi un déploiement de sujets variés, choisis par les internautes eux-mêmes, qui ouvrent une discussion sur le thème qui les intéresse, souvent des sujets caractéristiques de la religion, des questions inhérentes à la nature humaine et auxquelles la religion tente de répondre : la mort, la maladie, la vieillesse, les relations familiales... On constate que les sujets les plus prisés, plus que les discussions sur la conférence du dimanche, sont ceux de la rubrique "Vie de foi ", où sont abordés des thèmes de la vie quotidienne en lien avec la foi, et, plus particulièrement encore, l'homosexualité puis les femmes et leur place dans l'Église. Ces deux sujets sont souvent, d'après les messages, des pierres d'achoppement dans l'adhésion à l'Église dont l'attitude est parfois critiquée par des personnes se disant par ailleurs catholiques. On parle ainsi de "discrimination de l'Église à ne pas accorder le mariage aux homosexuels ", " problèmes de crédit de l'Église »... Selon Danièle Hervieu-Léger (2003), l'homosexualité est un enjeu majeur dans la modernité religieuse comme remise en cause de l' " ordre sacré » de la nature. Les messages du forum illustrent la tendance au « traitement compassionnel » ${ }^{22}$, où les hétérosexuels plaignent les homosexuels de leur situation délicate dans l'Église malgré leur foi qui serait tout aussi profonde et authentique. Mais ils ne sont pas traités comme des malades, bien au contraire : une émotion scandalisée s'empare du forum lorsqu'une personne parle de traitement médical. Sur

21. Nous pouvons citer un message de la rubrique «divorce» du forum qui illustre bien ce qu'est un «bricolage ": "Et si je puis encore aujourd'hui, à 52 ans, me reconnaitre CHRÉTIENNE, ce qui n'a rien à voir avec croyante et encore moins avec catholique, c'est parce que, dans ce que j'ai retenu des Évangiles (mais ai-je mal compris ?), il n'y a rien, absolument rien qui contredise mon humanisme "militant". Mais qu'est-ce que se reconnaitre chrétien quand on ne croit pas ? C'est tout simplement dire que le message évangélique est beau et encourageant, optimiste et bienveillant, dans son humanité et dans son accueil de tout ce qui est humain, le misérable et le faible comme le sublime et le fort. »

22. Expression d'Hélène Buisson-Fenet, citée par Danièle Hervieu-Léger (2003, p. 252). 
ce forum catholique, ceux qui soutiennent et osent rappeler la position de l'Église sont minoritaires. Les propos tenus ne s'inscrivent pas dans une fidélité absolue au dogme, montrant par conséquent comment chacun peut construire son propre système de croyance, avec d'autant plus de facilité que le contrôle sur Internet est quasi inexistant.

À partir de ce site, chacun peut aussi organiser la retraite de carême qu'il souhaite. C'est en quelque sorte une proposition de religion « à la carte ». Certes, les retraitants des abbayes sont libres de participer ou non aux offices, d'aller se promener, etc. mais la liberté de Retraite dans la Ville est encore plus grande puisqu'elle touche autant l'espace que le temps. En un sens, il n'y a plus aucune contrainte, ni de temps ni d'espace, imposée par la pratique de la religion et, ainsi, chaque individu peut « bricoler » sa pratique religieuse, écoutant les vêpres le matin et lisant la conférence du dimanche le mercredi. Sans compter, comme nous l'avons dit, que chacun peut adhérer à ce qu'il veut dans ce qu'on lui propose, sans contrôle d'aucune sorte. Si cette liberté qu'Internet introduit dans la pratique religieuse est l'un de ses meilleurs atouts pour permettre à ceux qui sont éloignés des pratiques institutionnelles d'en retrouver le chemin, elle peut néanmoins entraîner des pratiques qui ne pourraient avoir lieu dans l'espace institutionnel codifié et contrôlé car elles sont justement en marge de ce qui est reconnu officiellement. Les dominicains n'ont pas pour objectif d'accréditer ces «bricolages ", et s'ils le font, ce n'est que pour tenter ensuite de ramener les individus dans le cadre institutionnel de l'Église :

" Nous ne cherchons pas à nous adapter aux croyances multiples d'aujourd'hui, nous cherchons à dire la foi de l'Église, la parole de Dieu dans le langage d'aujourd'hui. Heu... Le noyau reste le même depuis 2000 ans, depuis que la foi de l'Église s'est construite, s'est formulée au quatrième ou cinquième siècle dans le langage qui était à l'époque celui de la culture grecque, de la culture latine. Ça, c'est plus audible pour les gens. Beaucoup de mots n'ont plus de sens. Heu... Parce que la culture a changé, le contenu reste le même, mais il doit être dit différemment, il doit être dit d'une manière différente. » (Frère Pascal)

Face aux bricolages des croyances, il s'agit donc de devenir plus compréhensible pour un public plus diversifié, mais le contenu doit pouvoir réunifier ces croyances autour du canon de l'Église. Dans cette perspective, l'un des atouts des dominicains est de ne pas être touchés par la perte de crédibilité de l'Église. Au contraire, les ordres religieux bénéficient aujourd'hui d'une meilleure reconnaissance de la société qui les perçoit comme témoignant par leur engagement total, et pas seulement en dictant des dogmes.

\section{Anonymat et désengagement religieux}

L'impersonnalité est l'un des risques majeurs de cette retraite puisque chacun la vit seul chez soi, et sous un pseudonyme lors des contacts avec les autres retraitants, notamment sur le forum, alors que parallèlement, la dimension 
communautaire est constitutive de la religion. C'est pourquoi les dominicains insistent sur l'importance de l'existence d'une communauté réelle derrière l'écran de l'ordinateur. Pour le frère Pascal, cette dimension est essentielle pour s'opposer à l'impersonnalité d'Internet, aux sites qui n'ont qu'un but informatif ou commercial :

«Internet n'est qu'un moyen. D'abord au sens où c'est porté par une communauté. C'est toute une communauté religieuse physique, donc c'est pas une communauté, on pourrait imaginer un projet comme ça, fait par 10 frères dans 10 couvents du monde différents, donc une communauté virtuelle qui s'adresserait à des gens. Ici ce n'est pas ça. Ici, c'est une communauté réelle qui porte le projet, c'est des frères qui vivent ensemble toute l'année et nous en discutons souvent au repas, nous sommes ensemble, nous prions ensemble. »

Pour cette retraite, les dominicains accueillent les retraitants dans leur communauté, par la pensée, la prière, comme ils accueillent réellement ceux qui viennent dans leur couvent. Cette présence réelle est illustrée par la présentation, avec photographie de chaque frère ou sœur qui participe au projet, hormis ceux et celles qui assurent l'accompagnement spirituel. La dimension communautaire et le lien social sont centraux dans la religion, nous ne pouvons imaginer de « cyberreligion » pure, à moins de revoir les définitions de l'objet (Durkheim, $1994)^{23}$. Cette dimension est très importante pour les personnes réellement touchées par la retraite, mais qui sont géographiquement ou matériellement éloignées des pratiques. Elles peuvent ainsi retrouver une certaine forme de communauté de croyance. Cela suppose aussi qu'une communauté élaborée sur le Net ne soit pas réellement une communauté religieuse. Celle qui se construit peu à peu entre les internautes, principalement ceux du forum, peut nous éclairer à ce sujet.

En vérité, cette communauté n'est, pour une bonne part, que virtuelle bien que, parfois, certains essaient de se voir physiquement. En premier lieu, les internautes ne se rencontrent pas autrement que par message sur Internet et, de surcroît, ne peuvent se connaître véritablement, à cause du pseudonyme sous lequel ils communiquent sur le forum. Certes, la lecture des messages, dans les différentes rubriques, permet de cerner de mieux en mieux qui est Passerlejourdain ou Mamipoule mais le nom, essentiel d'une identité, demeure absent. L'anonymat est ce que viennent chercher les internautes, pouvoir se révéler, parler de choses qui les touchent profondément sous couvert d'anonymat. Dans la rubrique « cancer », on peut lire en effet :

«Une confidence, puisque l'anonymat est préservé sur ce forum. »

23. Pour Durkheim, la communauté a une place importante dans sa définition de la religion : «Une religion est un système solidaire de croyances et de pratiques relatives à des choses sacrées, c'est-à-dire séparées, interdites, croyances et pratiques qui unissent en une même communauté morale, appelée Église, tous ceux qui y adhèrent ». 
Les pseudonymes choisis peuvent alors masquer complètement une identité, telle cette jeune fille au nom à particule qui s'inscrit au forum sous le nom de Robert $^{24}$. Cet anonymat, caractéristique d'Internet n'est-t-il pas, pourtant, dans un certain sens contraire à l'importance du nom dans la religion catholique ? Le frère Pascal, dans sa conférence sur l'apparition du Christ ressuscité à Marie-Madeleine, a justement souligné ce passage où Jésus appelle Marie par son prénom. La question qui se pose alors est celle de savoir si l'on peut considérer comme communauté religieuse une communauté virtuelle qui se vit dans l'anonymat.

Alors que la liberté de temps, d'espace et de degré d'engagement, notamment, semblait le déterminant de cette communauté sur Internet, l'exemple du Notre Père récité à 19 heures, montre au contraire le besoin de cadres pour essayer de se construire réellement comme communauté, avec des référents communs institutionnalisés d'une certaine manière. Or, ces cadres ne seront justement pas dans l'espace informatique, mais bien dans l'espace réel, qui retrouve la contrainte du temps, mais aussi d'engagement puisque chacun doit le réciter chaque jour à la même heure. Si une communauté religieuse peut s'élaborer à partir d'Internet, ce ne sera qu'un point de départ qui nécessitera des rencontres corporelles et des cadres qui viendront réduire cette liberté quasi totale du monde virtuel.

L'anonymat est omniprésent dans cette forme de pratique religieuse jusque dans les embryons de communauté qui se constituent. Or, la modernité religieuse se caractérise par la démonstration de l'engagement, la manifestation de ses croyances, comme le montrent les grands rassemblements telles les Journées Mondiales de la Jeunesse ou les pèlerinages comme celui de Saint-Jacques. Ce non-engagement des retraitants sur Internet est en contradiction avec les donnés du religieux moderne. Il n'y a finalement rien de plus discret que de pratiquer par Internet, utilisant un pseudonyme lorsqu'on est en relation avec d'autres. Or, on ne peut s'engager que personnellement, dans sa personne. C'est ainsi que se définit un engagement. Il ne peut y avoir de véritable engagement religieux sous anonymat. Et cette pratique semble paradoxalement attirer aujourd'hui alors que l'ère est à la démonstration. Ces deux tendances que l'on pourrait croire successives dans le temps coexistent donc. Nous pouvons apporter une tentative d'explication en reprenant la répartition par âge des participants à la retraite. Seuls 4,1\% avaient moins de 25 ans en 2006. Or, ce sont particulièrement les jeunes que l'on voit engagés dans les grands événements que nous citions, sans être pour autant des pratiquants réguliers. Il semble donc que ce ne soit pas ceux qui recherchent cet anonymat sur Internet, mais plutôt les personnes plus âgées ne se reconnaissant peut-être pas dans les formes expressives du religieux moderne. Cette pratique dénuée pratiquement des formes sensibles ne convient

24. Nous le savons par l'intermédiaire des dominicains qui, eux, peuvent savoir à quel nom réel correspond un pseudonyme. 
pas à ceux qui recherchent à la fois communauté de croyance et démonstration de l'engagement. Ceci expliquerait aussi le peu de participation des jeunes à cette retraite. En effet, pour ceux qui ne pratiquent pas régulièrement, une retraite sur Internet n'apporte pas les dimensions sensibles et finalement non religieuses qui les attirent vers les Journées Mondiales de la Jeunesse. La religion nue, dénuée d'autres agréments ne répond pas aux besoins des jeunes peu engagés dans l'Église, mais bien à ceux qui sont déjà impliqués et qui voudront justement bénéficier de cet espace en dehors du sensible, nous y reviendrons.

\section{Une religiosité virtuelle?}

La question de la virtualité se pose dans toute son acuité dans le cas de l'accompagnement spirituel en ligne que propose aussi Retraite dans la Ville. Il soulève nombre de questions sur sa légitimité aux dominicains eux-mêmes ${ }^{25}$. Nous faisons nôtre cette interrogation du frère Xavier Loppinet : "Un accompagnement spirituel “vrai” peut-il avoir lieu sur Internet?» Plusieurs éléments sont à considérer : la pertinence d'un accompagnement "à domicile », le double anonymat de l'accompagnateur et de l'accompagné, la virtualité de la rencontre.

L'accompagnement en ligne, tout comme les offices et autres éléments de ce site, permet à des individus éloignés géographiquement ou à cause d'un handicap d'en bénéficier, alors que cela leur est habituellement impossible. Le fait qu'il puisse se faire "à domicile ", sans démarche particulière, ouvre aussi cette possibilité à des gens qui n'oseraient pas entrer dans une église ou un presbytère. En cela, cette pratique peut paraître plutôt favorable à un renouveau de l'accompagnement spirituel ${ }^{26}$.

Mais contrairement à d'autres sites, l'accompagnement de Retraite dans la Ville est anonyme et aucun des deux acteurs de la relation ne choisit l'autre. Ainsi, comme le souligne le frère Pascal, l'accompagnateur répond à des questions et non à des personnes. Mais quelle valeur peut avoir l'impersonnalité et l'anonymat dans la pratique religieuse et encore plus dans une religion de l'appel ? Peut-on dire qu'il s'agit d'une véritable relation, comme le sous-entend habituellement l'accompagnement entre deux personnes, s'il n'y a plus que des questions et des réponses dénuées de tout autre référent ? Enfin, cette relation, s'il y en a une, est purement virtuelle, en plus d'être impersonnelle, c'est-à-dire qu'elle n'est pas complètement réelle au sens où les individus ne se rencontrent

25. En référence à un article de Xavier Loppinet dans Lumière et Vie, 268 : «L'accompagnement spirituel sur Internet est-il possible ? »

26. Pour cet élément de la retraite, le but de rejoindre les éloignés semble en grande partie atteint si l'on en croit Xavier Loppinet: «De fait, les personnes qui demandaient un accompagnement exprimaient toutes une forme d'isolement, au moins psychologique. Deux accompagnateurs, lors du bilan, ont employé l'expression de "bouteille à la mer" jetée par leur correspondant sur le net. ", op. cit. 
pas, et le risque potentiel est celui d'une certaine facilité que nous allons aborder ci-après.

Il faudrait distinguer, dans cette retraite, ce qui relève du virtuel et de la réelle expérience religieuse, distinction difficile à faire. Les deux dimensions sont imbriquées : pour la méditation quotidienne, la prière de l'individu peut être réelle, mais le lien à une communauté religieuse est virtuel. Par ailleurs, pour tenter de détourner le danger d'une relation qui s'établirait durablement dans le virtuel, il est clairement affirmé, à plusieurs reprises, que l'accompagnement spirituel ne peut durer que le temps du carême. À l'issue de cette retraite, les individus sont éventuellement orientés vers un accompagnement traditionnel. Car, pour le Frère Pascal, " nous ne sommes pas destinés à vivre dans le virtuel ». Le risque de vouloir aller plus loin dans la pratique religieuse par Internet n'est cependant pas absent ; après les offices et l'accompagnement en ligne, pourquoi ne pas imaginer des sacrements en ligne ? L'Église rejette catégoriquement cette idée qui va à l'encontre de l'essence même du sacrement où le corps, dans les gestes et la rencontre des personnes, a une place prépondérante. Mais la question n'est pas si incongrue si l'on en croit l'article de Xavier Loppinet : "Plusieurs accompagnateurs ont dit avoir eu des demandes de confessions auxquelles ils n'ont, bien sûr, pas répondu autrement que par le renvoi à une rencontre réelle avec un prêtre. »

Le virtuel, dans ce cas, est en fait associé à la facilité, facilité de faire et dire dans un milieu qui ne serait pas vraiment le réel et qui, par-là, n'aurait pas de véritables conséquences pour le réel. Et c'est contre ce genre de facilité que les dominicains s'élèvent. La religion, bien que visant un au-delà du réel, ne veut pas avoir parti lié avec le virtuel. Cette facilité se trouve confirmée au regard des nombreux accompagnés qui ont cessé l'échange sans en avertir l'accompagnateur. On peut en effet citer Xavier Loppinet : "Beaucoup d'accompagnateurs ont été surpris de la facilité avec laquelle leurs correspondants abandonnaient du jour au lendemain, sans explication, les échanges ${ }^{27}$. Il est simple, lorsqu'on ne voit pas la personne, qu'on ne la verra jamais et qu'elle ne sait pas qui on est, de rompre rapidement ou brusquement la relation. Par conséquent, la religion devient alors désengagée puisque aucun lien ne retient l'individu à la communauté.

Condamner la virtualité qui serait étrangère au monde, à la réalité est un topos associé aux critiques faites à Internet. Mais à bien y réfléchir, la virtualité n'est peut-être pas si étrangère à la religion. Parler à quelqu'un que l'on ne voit pas, que l'on ne connaît pas, que l'on ne rencontre pas, c'est le principe de toute prière à une divinité ou à des saints. Par exemple, les questions posées à l'accompagnateur spirituel en ligne se rapprochent d'une demande faite à Dieu, ou à un saint qui répond de manière évasive, surtout lorsque l'échange ne dure

27. Note de l'auteur : «Une partie importante abandonnait (43\%), quand même la relation semblait établie. » Loppinet, op. cit. 
que le temps d'un message. Ce rapport à Internet rappellerait la relation à un suprasensible. La religion sur Internet évacue, pour une part au moins, le sensible. Le message religieux ne devient que du message, c'est le cas pour l'accompagnement spirituel où le frère répond à une question et non à une personne. C'est comme s'il n’y avait plus que du fond, dénué de forme sensible. Mais dans ce cas, pourquoi condamner le virtuel ? Nous pouvons nous référer, par exemple, à l'ouvrage d'A. Kleinberg (2005) qui expose comment l'Église, devant l'engouement pour les cultes de saints au IV ${ }^{\mathrm{e}}$ siècle, les a institutionnalisés pour mieux les contrôler. Tout ce qui touche à une pratique suprasensible, d'un individu particulier à une entité divine ne peut pas être contrôlé. Or, l'Église veut contrôler les pratiques, les codifier, pour se construire en tant qu'Église, ce qui ne peut pas être fait dans ce cas, ainsi que le prouvent les discussions sur le forum, à l'écart des dogmes. Ainsi, Internet peut-il recréer une relation directe de l'individu à un suprasensible.

\section{Quel avenir pour la retraite sur Internet ?}

À l'encontre de cette virtualité, le défi des dominicains est de faire d'Internet un lieu d'authenticité religieuse. Par deux fois, ce terme est employé dans le dossier de presse.

"Un désir authentique de spiritualité est ainsi exprimé sur Internet. »(p. 2)

«La retraite sur Internet ne dure pas l'espace d'un clic ou d'un message envoyé, mais se veut fondatrice d'une expérience spirituelle authentique. » (p. 7)

L'ordinateur peut en effet devenir le lieu d'une pratique religieuse, selon des témoignages de retraitants, tel l'exemple des bougies que nous évoquions. Cela montre que dans la modernité religieuse, il n'y a plus vraiment de lieu spécifique du religieux, qui peut se vivre partout. De même que les églises servent aussi pour des concerts et diverses activités non religieuses. Cette retraite sur Internet peut être dite authentique s'il n'y a plus, en amont, de lieux sacrés où se jouerait nécessairement et exclusivement la pratique religieuse. À partir de ce moment, tout lieu peut devenir celui d'une pratique religieuse " authentique ». Si les dominicains insistent sur le fait que cet événement ne dure que le temps du carême, c'est sans doute parce qu'ils considèrent qu'une pratique religieuse sur Internet dans la durée ne serait pas vraiment authentique. Dans ces conditions, une certaine ambiguïté de leur part subsiste dans cette qualification.

Si Internet ne doit pas remplacer une pratique religieuse " réelle ", c'est parce qu'il ne peut pas tout donner de la religion, les sacrements notamment. Les dominicains ne conçoivent pas Internet comme un outil de remplacement de la pratique religieuse, l'assistance aux vêpres en ligne dispensant de se rendre dans une église, par exemple. Cette position est précisée dans le dossier de presse : «La fréquentation quotidienne d'un site Internet ne saurait remplacer complètement une retraite faite de silence et de déplacements ». Plutôt qu'un remplacement, 
Internet peut donc servir de lancement ou de renouveau de la pratique religieuse pour ceux qui l'avaient peu à peu abandonnée. Pour le frère Pascal :

"Ensuite c'est une démarche que les gens initient et qu'ils poursuivent eux-mêmes, chez eux, dans leur paroisse, dans les groupes qu'ils fréquentent, heu donc, le but, c'est d'aider les gens."

Internet n'est pas une fin en soi, ce qui signifie qu'il n'est pas considéré comme le lieu d'une authenticité spirituelle durable. Comme le dit le frère Pascal, Internet n'est qu'un moyen, un moyen d'atteindre les gens, mais il ne saurait représenter une pratique religieuse par lui-même. C'est l'une des raisons pour lesquelles Retraite dans la Ville n'est qu'un événement ponctuel.

Devant le succès de cette formule, même si le public attendu n'est pas celui réellement présent, nous pouvons nous demander si les dominicains envisagent une suite possible pour le reste de l'année. Malgré leur réticences à pérenniser une pratique religieuse virtuelle, il semblerait que la mise en ligne des offices, qui offre à des personnes isolées et dans l'impossibilité matérielle de bénéficier des services de l'Église, la possibilité de voir et de prier lors d'un office communautaire, puisse être proposée à l'année. Cela ne serait pas spécifique à Internet mais rejoindrait le même principe que la messe télévisée. La question centrale demeure celle du public visé initialement : les spirituellement éloignés placés en tête de la mission. Sans doute les dominicains devront-ils travailler plus précisément sur les résultats des sondages concernant ce que les quelques personnes éloignées de l'Église viennent chercher et trouvent dans cette retraite pour parvenir à les attirer plus massivement.

\section{Conclusion}

Si le chantier reste encore à poursuivre sur ce sujet, nous pouvons déjà dire que Retraite dans la Ville illustre parfaitement les différentes modalités de la modernité religieuse concernant le catholicisme occidental. En réaction à la perte des pratiques religieuses, par éloignement spirituel de l'Église de la part des individus ou géographique du fait de la baisse du nombre de prêtres en lien avec la baisse des vocations, les dominicains utilisent un outil qui, niant l'espace et le temps, peut atteindre un public vaste et diversifié. Mais parce que la liberté des pratiques qu'Internet permet, récuse l'idée d'un temps et d'un espace religieux, la pratique religieuse en ligne, bien que présentée comme authentique par les organisateurs, reste incomplète. Il y manque des éléments essentiels qui demeurent spatialisés et temporalisés, comme les sacrements. Par ailleurs, la virtualité, bien que non étrangère à la religion, ne saurait faire partie institutionnellement de l'Église : elle ne peut faire l'objet d'aucun contrôle. Enfin, le but recherché: toucher les gens en marge de l'Église, reste encore à atteindre, la grande majorité des retraitants étant déjà pratiquants. Plutôt que de toucher les populations utilisatrices d'Internet comme il était escompté, ce sont bien les catégories identifiées 
comme déjà pratiquantes qui participent à cette retraite. Celle-ci pose par conséquent la question de la pertinence d'un médium, d'un outil à distance pour diffuser la religion à des catégories particulières, car il est impossible d'être sûr que cela arrivera réellement aux personnes déterminées (contrairement à la mission directe par exemple).

Toutes ces questions ne sont pas spécifiques à Retraite dans la Ville, mais aussi à toutes les formes de religion sur Internet qui fleurissent actuellement. La particularité de cet événement est de vouloir proposer un service religieux réel, normalement institutionnalisé, et pas simplement un site de spiritualité, d'aide à la prière comme il y en a tant.

D'autre part, cette retraite à domicile pose la question de l'extramondanité de la religion catholique et de ses pratiques. Il semble en effet que l'extramondanité ait perdu sa signification religieuse ; les dominicains ont donc opté pour intramondaniser une pratique à l'origine proprement hors du monde. Ainsi, la formule classique de Sebastian Franck pour l'ascèse intramondaine protestante : « Tu croyais avoir échappé au monastère, mais désormais chacun doit être moine sa vie durant » peut alors se traduire d'une autre manière : " tu croyais avoir échappé à la retraite en monastère, tu peux désormais la faire dans ta vie quotidienne » (Weber, 1991 : 382).

Isabelle JONVEAUX EHESS Paris, Università degli Studi di Trento isabelle.jonveaux@laposte.net

DuRKheIm Émile, 1994, Les Formes élémentaires de la vie religieuse, Paris, PUF-Quadrige. Hervieu-Léger Danièle, 2003, Catholicisme, la fin d'un monde, Paris, Bayard.

-, 1986, Vers un nouveau christianisme ?, Paris, Éditions du Cerf.

ISAMBERT François-André, 1976, "La sécularisation interne du christianisme ", Revue

Française de Sociologie, XII, pp. 573-589.

Kleinberg Aviad, 2005, Histoires de saints, leur rôle dans la formation de l'Occident

(trad. Moshé Méron), Paris, NRF-Gallimard.

LOPPINET Xavier, "L'accompagnement spirituel sur Internet est-il possible ? », Lumière et Vie, 268, $\mathrm{n}^{\circ}$ spécial L’Église dans la culture médiatique.

Pelletier Denis, 1997, Les Catholiques en France depuis 1815, Paris, La Découverte.

Weber Max, 1991, Histoire économique, Paris, NRF-Gallimard, coll. «Bibliothèque des

Sciences Humaines ».

-, 2003, L'Éthique protestante et l'esprit du capitalisme (trad. Isabelle Kalinowski), Paris, Gallimard, coll. «Tel». 
i76 - ARChives De SCIENCES SOCIALES DES RELIGIONS

\section{Résumé}

Retraite dans la Ville, retraite de carême sur Internet organisée par les dominicains, illustre bien des aspects de la modernité religieuse. Alors que le but premier de cette retraite était d'attirer ceux qui ne pratiquent pas ou plus, particulièrement les jeunes familiarisés avec Internet, ce sont plutôt les catégories déjà pratiquantes qui participent à cet événement. Ce site qui apporte la religion "à domicile" pose la question de l'extramondanité de la religion; si les dominicains, intramondanisent une pratique est-ce parce que toute sortie du monde serait devenue impossible ou sans attrait? On assiste alors à l'élaboration de pratiques religieuses sur Internet caractérisées par l'anonymat et le désengagement. Internet introduit aussi une dimension virtuelle qui, sans être totalement étrangère à la religion, est condamnée par les autorités religieuses pour la facilité qu'elle entraîne et son absence de contrôle qui peut renforcer le "bricolage" des croyances.

Mots-clés: Modernité religieuse, Internet, extramondanité, bricolage des croyances, virtualité.

\section{Abstract}

For instance of religion in the modern age, we can study a suggest of retreat on Internet, Retraite dans la Ville, at the time of Lent. This event is organized by French Dominicans who hope that in this way they get in touch with individuals who otherwise would not make a retreat. But in fact, participants belong to categories of churchgoers and not these of the Net surfers. This retreat poses problem of the "out of the worldliness" of Catholicism which seems no longer attractive and the Dominicans are not trying to reverse this tendency. On this website religious practices are elaborated, characterised by easiness and anonymity. Although virtuality is not as remote from religion as it might seem to be, it should not become a part of the institutional practice of the Church, because it cannot be controlled and cannot provide the whole extent of religious practice, and it can confirm "patching together of beliefs".

Key words: Religious modernity, Internet, extramondanity, believing's bricolage, virtuality.

\section{Resumen}

Retiro en la Ciudad, retiro de Cuaresma en Internet organizado por los dominicanos, ilustra bien ciertos aspectos de la modernidad religiosa. Mientras que el objetivo principal de este retiro era el de atraer a aquellos que no practican o han dejado de practicar, a los jóvenes particularmente, familiarizados con Internet, son más bien las categorías ya practicantes quienes participan de este hecho. Este sitio, que lleva la religión "a domicilio", plantea la cuestión de la extramundanidad de la religión; si los dominicanos intramundanizan una práctica, ¿ es porque toda salida del mundo se habría vuelto imposible o sin atractivo? Asistimos entonces a la elaboración de prácticas religiosas en Internet caracterizadas por el anonimato y la falta de compromiso. Internet introduce también una dimensión virtual que, sin ser totalmente extranjera a la religión, es condenada por las autoridades religiosas por la facilidad que conlleva y por su ausencia de control que puede reforzar el "bricolage" de las creencias. (Trad. de Véronica Béliveau-Giménez)

Palabras clave: Modernidad religiosa, Internet, extramundanidad, bricolage de las creencias, virtualidad. 